\title{
Perfil sociodemográfico, epidemiológico e comportamental de mulheres infectadas pelo HTLV-1 em Salvador-Bahia, uma área endêmica para o HTLV
}

\author{
Sociodemographic, epidemiological and behavioral profile of women infected \\ with HTLV-1 in Salvador, Bahia, an endemic area for HTLV
}

\author{
Ivanoska Moxoto ${ }^{1}$, Ney Boa-Sorte ${ }^{1,2}$, Ceuci Nunes ${ }^{1}$, Augusto Mota ${ }^{1,2}$, \\ Alexandre Dumas ${ }^{1}$, Inês Dourado ${ }^{3}$ e Bernardo Galvão-Castro ${ }^{1,2}$
}

\begin{abstract}
RESUMO
O objetivo foi descrever as características sociodemográficas, epidemiologicas e comportamentais de mulheres infectadas (64) e não infectadas (66) pelo HTLV-1 em Salvador, Babia. O diagnóstico sorológico foi obtido por ELISA, WB e Imunofluorescência. Dados epidemiologicos e sociodemográficos foram coletados utilizando questionário padronizado. O Qui-quadrado ou teste de Fisher foi usado para dados categóricos eANOVA ou Kruskall Wallis (3 grupos) e teste Tou Mann Whitney (2 grupos) para os dados contínuos. As variáveis associadas foram ajustadas por regressão logística. Mais da metade $(57,8 \%)$ das mulheres soropositivas eram assintomáticas. As sintomáticas (com PET/MAH) tinham menor escolaridade. A comparação entre mulheres soropositivas e soronegativas mostrou que a hemotransfusão, a prática de sexo anal, coitarca antes dos 18 anos e ter mais de 3 parceiros sexuais na vida foram fatores de risco para infecção pelo HTLV-1. A prevenção da transmissão sexual e vertical (aleitamento materno) deve ser reforçada e triagem no pré-natal mandatória.
\end{abstract}

Palavras-chaves: HTLV-1. Fatores de risco em mulheres.

\begin{abstract}
The objective was to describe the sociodemographic, epidemiological and behavioral characteristics of women infected with HTLV-1 (64) and uninfected women (66) in Salvador, Babia. The serological diagnosis was obtained via ELISA, Western Blot and Immunofhorescence. Epidemiological and sociodemographic data were collected using a standardized questionnaire. The chi-squared or Fisher test was used for categorical data and ANOVA or Kruskal-Wallis (3 groups) and the T-test or Mann-Whitney (2 groups) were used for continuous data. Associated variables were adjusted using logistic regression. More than half (57.8\%) of the seropositive women were asymptomatic. The symptomatic women (with HAM/TSP) had fewer years of education. Comparison between seronegative and seropositive women showed that blood transfusion, anal sex practices, first sexual intercourse before the age of 18 years and three or more sexual partners over women's lifetime were risk factors for HTLV-1 infection. The prevention of both sexual transmission and vertical transmission (breastfeeding) should be reinforced. Prenatal screening is of paramount importance.
\end{abstract}

Key-words: HTLV-1. Risk factors among women.

0 vírus linfotrópico da célula T humana tipo 1 (HTLV-1) está diretamente associado a pelo menos três patologias: leucemia/ linfoma de células T do Adulto (LLTA) ${ }^{21620}$; paraparesia espástica tropical/mielopatia associada ao HTLV-1 (PET/MAH) ${ }^{614}$ e uveíte associada ao HTLV-1(UAH) ${ }^{11}$.
Este vírus é transmitido por três vias: sexual, com cerca de $60 \%$ de eficiência do homem para mulher e, no sentido inverso, em torno de 4\%; sangüínea, através do compartilhamento de seringas e/ou agulhas contaminadas ou transfusão de sangue, e vertical, da mãe para o filho, principalmente pelo aleitamento

\footnotetext{
1. Escola Bahiana de Medicina e Saúde Pública, Fundação Bahiana para o Desenvolvimento das Ciências, Salvador, BA, Brasil. 2. Laboratório Avançado de Saúde Pública, Centro de Pesquisas Gonçalo Moniz, Fundação Oswaldo Cruz, Salvador, BA, Brasil. 3. Instituto de Saúde Coletiva, Universidade Federal da Bahia, Salvador, BA.

Apoio financeiro: Fundação de Amparo a Pesquisa do Estado da Bahia (FAPESB); Secretaria de Saúde do Estado da Bahia (SESAB) e Conselho Nacional de Desenvolvimento Científico e Tecnológico (CNPq)

Address to: Dr. Bernardo Galvão-Castro, LASP/CPqGM/FIOCRUZ. R. Waldemar Falcão 121, Candeal, 40295-001 Salvador BA, Brasil

Tel: 55 71 3176-2213

e-mail: bgalvao@cpqgm.fiocruz.br

Recebido para publicação em 22/6/2006

Aceito em 19/1/2007
} 
materno ${ }^{17}$. Estima-se que 15 a 20 milhões de pessoas estejam infectadas pelo HTLV-1 no mundo ${ }^{4}$. 0 Japão, Caribe, América do Sul e Central, África Equatorial, Oriente Médio e Melanésia são as principais áreas endêmicas mas a prevalência nessas áreas, não é uniforme, variando de uma cidade para outra ${ }^{17}$.

O HTLV-1 foi identificado pela primeira vez no Brasil em 1986, entre imigrantes japoneses provenientes de Okinawa, na Cidade de Campo Grande, Mato Grosso do Sul ${ }^{9}$. Este vírus e suas patologias associadas estão amplamente descritos em diferentes regiões geográficas no Brasil, sendo Salvador a cidade brasileira com maior prevalência de HTLV-15.

Um estudo com amostra representativa da população geral de Salvador, demonstrou uma prevalência de 1,7\% (homens: $1,2 \%$; e mulheres: $2 \%$ ), observando-se um aumento significativo da prevalência com a idade, principalmente no sexo feminino, atingindo $9 \%$ em mulheres acima de 51 anos $^{3}$.

Apesar da prevalência mais elevada do HTLV-1 em mulheres, os estudos envolvendo este grupo são escassos. Ademais, não existe até o momento, nenhum estudo específico sobre as características sociodemográficas, epidemiológicas e comportamentais desta infecção no sexo feminino em nosso meio. Este trabalho descreve estas características em mulheres infectadas pelo HTLV-1, atendidas no Centro Integrativo de HTLV-1 e hepatites virais (CIHHV) da Escola Bahiana de Medicina e Saude Pública (EBMSP), Fundação Bahiana para Desenvolvimento das Ciências (FBDC).

\section{MATERIAL E MÉTODOS}

Desenho, local, amostra e aspectos éticos do estudo. Trata-se de um estudo descritivo de dois grupos de mulheres cuja principal característica de diferenciação foi a positividade sorológica para o HTLV-1. 0 estudo foi aprovado pelo Comitê de Ética em Pesquisa da FBDC e realizado no período de março a agosto de 2004 no CIHHV, situado no Centro Médico Docente Assistencial de Brotas (CMDAB), centro de referência no Estado da Bahia para a assistência de indivíduos infectados pelo HTLV-1 e 2. Este centro oferece assistência integral e interdisciplinar ao portador do HTLV.

As mulheres atendidas no CIHHV com idade superior a 14 anos somavam 145 pacientes no início da pesquisa. Deste total, 74 pacientes, que compareceram e concordaram em participar voluntariamente, após assinatura do Termo de Consentimento Livre e Esclarecido (TCLE), foram incluídas no estudo. Pacientes gestantes e que tinham sorologia positiva para HIV ou HTLV-2 foram excluídas, perfazendo um total de 64 pacientes soropositivas estudadas. Um grupo de 71 mulheres soronegativas para o HTLV-1 que procuraram o serviço de ginecologia do $\mathrm{CMDAB}$, durante 0 período de realização do estudo, foram selecionadas para fins de comparação com o grupo de mulheres positivas. Destas, cinco foram excluídas (três não concluíram as avaliações, uma estava gestante e outra se recusou a realizar as sorologias), totalizando 66 pacientes soronegativas.
Todas as pacientes foram entrevistadas utilizando-se um questionário padronizado com questões relativas a aspectos sociodemográficos (idade, escolaridade, renda familiar, estado civil), epidemiológicos (transfusão de sangue, uso de drogas, doenças sexualmente transmissíveis, ter sido amamentado) e comportamentais (práticas sexuais, uso de preservativos, número de parceiros sexuais na vida, idade da coitarca).

Métodos laboratoriais. Todas as pacientes realizaram sorologia para HTLV e HIV. As amostras de plasma foram triadas para HTLV-1 e 2 (HTLV-I/HTLV-II Ab-Capture ELISA Test System) e HIV-1 e 2 (HIV-1/HIV-2 Ab-Capture ELISA Test System) ambos da Ortho® Clinical Diagnostic Inc., Raritan, New Jersey, USA). Os resultados repetidamente positivos testados em duplicata foram submetidos a teste confirmatório. A confirmação, para o HIV, foi realizada com imunofluorescência indireta (Biomanguinhos, FIOCRUZ, Rio de Janeiro, Brasil), e, para o HTLV, a confirmação e a diferenciação entre HTLV-1 e HTLV-2 foi realizada com 0 Western Blot (HTLV Blot 2.4. Genelabs, Singapure). Para as amostras com reação indeterminada para o HTLV, foi realizada uma reação em cadeia de polimerase (PCR).

Análise dos dados. As pacientes estudadas foram divididas em 3 grupos: HTLV negativas, HTLV positivas assintomáticas e as HTLV positivas sintomáticas (presença de PET/MAH ou LLTA). Para a análise de fatores sabidamente associados ao HTLV, as pacientes HTLV positivas foram reagrupadas em um único grupo. Na análise univariada, para comparação das variáveis sociodemográficas e epidemiológicas entre os grupos, utilizou-se a Análise de Variância (ANOVA) ou o teste não-paramétrico de Kruskal-Wallis. Dados categóricos foram analisados com o teste do qui-quadrado (com ou sem correção de Yates) ou com o teste exato de Fisher. 0 nível de significância estatística estabelecido foi de 0,05 . Na comparação entre os grupos HTLV positivos e negativos, as razões de chance não ajustadas (ORs) com os intervalos de confiança de $95 \%$ (IC95\%) foram calculadas em tabelas 2 x 2 medindo a associação entre a infecção por HTLV e as variáveis de interesse. Regressão logística foi realizada para ajustar as estimativas dos potenciais fatores associados. A análise foi realizada utilizando os softwares SPSS para a Windows ${ }^{\circledR}$ (versão 9.0) e Epi Info ${ }^{\circledR}$ para Windows ${ }^{\circledR}$ (versão 2000).

\section{RESULTADOS}

0 grupo infectado pelo HTLV-1 foi constituído por 64 mulheres (soropositivas) e, o de soronegativas por 66 pacientes. Entre as pacientes infectadas pelo HTLV-1, 37 (57,8\%) não apresentavam achados clínicos compatíveis com PET/MAH ou LLTA (denominadas assintomáticas) e 27 (42,1\%) tinham diagnóstico de PET/MAH (sintomáticas). As características sociodemográficas dos grupos de mulheres estudadas estão descritas na Tabela 1.

Todos as mulheres tinham renda familiar semelhante, porém houve diferença entre os grupos em relação a escolaridade 
$\left(\chi^{2}=5,97 ; \mathrm{p}=0,05\right)$, sendo que a menor escolaridade (até 8 anos de estudo) foi mais comum entre as mulheres sintomáticas em relação as assintomáticas e soronegativas. Em relação ao estado civil referido, observou-se uma maior proporção de viúvas entre as sintomáticas (com PET/MAH) em relação às assintomáticas (sem PET/MAH) e as soronegativas $(29,6 \%$ x 5,4\% x 6,1\%; $\mathrm{p}=0,012)$. A maioria $(63 \%)$ das pacientes com PET/MAH tinha idade superior a 50 anos. A média de idade neste grupo foi de 54,2 $\pm 12,7$ anos, enquanto nos grupos de infectadas assintomáticas e soronegativas foram respectivamente $42,5 \pm 14,4$ e 42,2 $\pm 14,7$ $\operatorname{anos}(\mathrm{F}=7,416 ; \mathrm{p}=0,01)$.

As características epidemiológicas e comportamentais para os três grupos foram descritas na Tabela 2. Na Tabela 3, as mesmas características são descritas através da comparação entre as mulheres infectadas pelo HTLV-1 e soronegativas. Idade da primeira relação sexual (coitarca), número de parceiros sexuais na vida, presença de DST e transfusão sanguínea foram as variáveis que apresentaram diferença significativa entre os três grupos avaliados (Tabela 2). Na comparação entre os grupos de soropositivas e soronegativas (Tabela 3 ), as práticas sexuais anais foram associadas a soropositividade para HTLV-1 $(p=0,025)$, enquanto a presença de DST não permaneceu significante $(\mathrm{p}=$ $0,08)$. No modelo multivariado contendo as variáveis associadas na análise univariada e as de plausibilidade biológica, obteve-se significância para a presença de hemotransfusão, presença de prática sexual anal, coitarca antes dos 18 anos e mais de três parceiros sexuais na vida (Tabela 4).

Tabela 1 - Comparação das características sociodemográficas entre mulberes infectadas pelo HTLV-1 e soronegativas atendidas no Centro Integrativo de HTLV-1 e hepatites virais/Centro Médico Docente Assistencial / Escola Babiana de Medicina e Saúde Pública.

\begin{tabular}{|c|c|c|c|c|c|c|c|}
\hline \multirow[b]{3}{*}{ Variável } & & & \multicolumn{4}{|c|}{ HTLV-1 ${ }^{+}$} & \multirow{3}{*}{$\mathrm{p}$} \\
\hline & \multicolumn{2}{|c|}{ HTLV-1 ${ }^{-}$} & \multicolumn{2}{|c|}{ assintomáticas* } & \multicolumn{2}{|c|}{ sintomáticas } & \\
\hline & $\mathrm{n}^{0}$ & $\%$ & $\mathrm{n}^{0}$ & $\%$ & $\mathrm{n}^{0}$ & $\%$ & \\
\hline Idade (anos) & & & & & & & 0,007 \\
\hline$<50$ & 46 & 69,7 & 26 & 70,3 & 10 & 37,0 & \\
\hline$\geq 50$ & 20 & 30,3 & 11 & 29,7 & 17 & 63,0 & \\
\hline Escolaridade (anos) & & & & & & & 0,05 \\
\hline$\leq 8$ & 36 & 54,5 & 18 & 48,6 & 21 & 77,8 & \\
\hline$>8$ & 30 & 45,5 & 19 & 51,4 & 6 & 22,2 & \\
\hline Renda (SM) ${ }^{* * *}$ & & & & & & & 0,727 \\
\hline$<1$ & 22 & 33,3 & 11 & 29,7 & 12 & 44,5 & \\
\hline 1 a 3 & 28 & 42,4 & 19 & 51,4 & 9 & 33,3 & \\
\hline 3 a 5 & 8 & 12,1 & 5 & 13,5 & 4 & 14,8 & \\
\hline$\geq 5$ & 8 & 12,1 & 2 & 5,4 & 2 & 7,4 & \\
\hline Estado Civil & & & & & & & 0,012 \\
\hline solteira & 21 & 31,8 & 8 & 21,6 & 5 & 18,5 & \\
\hline casada/amasiado & 37 & 56,1 & 21 & 56,8 & 11 & 40,7 & \\
\hline separada/divorciada & 4 & 6,1 & 6 & 16,2 & 3 & 11,1 & \\
\hline viúva & 4 & 6,1 & 2 & 5,4 & 8 & 29,6 & \\
\hline
\end{tabular}

*pacientes sem PET/HAM

***SM = Salários-mínimos
Tabela 2 - Comparação das características epidemiológicas e comportamentais entre mulberes infectadas pelo HTLV-1 e soronegativas atendidas no Centro Integrativo de HTLV-1 e Hepatites Virais/Centro Médico Docente Assistencial/Escola Bahiana de Medicina e Saúde Pública.

\begin{tabular}{|c|c|c|c|c|c|c|c|}
\hline \multirow[b]{3}{*}{ Variável } & & & \multicolumn{4}{|c|}{ HTLV-1 ${ }^{+}$} & \multirow{3}{*}{$\mathrm{p}$} \\
\hline & \multicolumn{2}{|c|}{ HTLV-1 $^{-}$} & \multicolumn{2}{|c|}{ assintomáticas* } & \multicolumn{2}{|c|}{$\underline{\text { sintomáticas }}$} & \\
\hline & $\mathrm{n}^{0}$ & $\%$ & $\mathrm{n}^{0}$ & $\%$ & $\mathrm{n}^{\circ}$ & $\%$ & \\
\hline Idade da coitarca (anos) & & & & & & & 0,008 \\
\hline$\leq 18$ & 28 & 43,8 & 27 & 75,0 & 17 & 63,0 & \\
\hline$>18$ & 36 & 56,3 & 9 & 25,0 & 10 & 27,0 & \\
\hline Parceiros sexuais navida $\left(\mathrm{n}^{\circ}\right)$ & & & & & & & $<0,001$ \\
\hline$>3$ & 8 & 12,1 & 20 & 54,1 & 10 & 37,0 & \\
\hline$\leq 3$ & 58 & 87,9 & 17 & 45,9 & 17 & 63,0 & \\
\hline \multicolumn{8}{|l|}{ Práticas sexuais anais ${ }^{1}$} \\
\hline $\operatorname{sim}$ & 12 & 18,8 & 14 & 38,9 & 9 & 33,3 & 0,072 \\
\hline não & 52 & 81,2 & 22 & 61,1 & 18 & 66,7 & \\
\hline \multicolumn{8}{|l|}{ Uso de preservativos ${ }^{1}$} \\
\hline raramente/não usa & 53 & 82,8 & 31 & 86,1 & 25 & 92,6 & 0,473 \\
\hline freqüentemente & 11 & 17,2 & 5 & 13,9 & 2 & 7,4 & \\
\hline \multicolumn{8}{|l|}{ História de DST ${ }^{2}$} \\
\hline $\operatorname{sim}$ & 6 & 10,2 & 3 & 13,0 & 6 & 37,5 & 0,025 \\
\hline não & 53 & 89,8 & 20 & 87,0 & 10 & 62,5 & \\
\hline
\end{tabular}

História de

transfusão sangüínea ${ }^{3}$

\begin{tabular}{lrrrrrrr}
$\operatorname{sim}$ & 5 & 76,9 & 9 & 25,7 & 7 & 28,0 & \\
não & 60 & 23,1 & 26 & 74,3 & 18 & 72,0 & \\
\hline Uso de drogas ilícitas & & & & & & & 0,686 \\
$\quad$ sim & 1 & 1,5 & 1 & 2,8 & 0 & 0,0 & \\
não & 65 & 98,5 & 36 & 97,2 & 27 & 100,0 & \\
\hline Foi amamentado & & & & & & & 0,084 \\
$\quad$ sim & 49 & 96,1 & 28 & 93,3 & 19 & 79,2 & \\
não & 2 & 3,9 & 2 & 6,7 & 5 & 20,8 & \\
\hline
\end{tabular}

'Três pacientes referiram não ter tido relações sexuais, sendo excluídas da análise ( 2 no grupo negativo e uma no grupo positivo assintomático). ${ }^{2}$ História de DST. O cálculo do qui-quadrado foi realizado sem considerar a resposta "não sabe/não se aplica". ${ }^{3}$ História de Transfusão sangüínea. Cinco pacientes referiram não saber se receberam hemotransfusão, sendo excluídas da análise (uma no grupo negativo e 4 no grupo positivo)

Tabela 3 - Comparação das características epidemiológicas e comportamentais de mulheres infectadas pelo HTLV-1 e soronegativas atendidas no Centro Integrativo de HTLV-1 e Hepatites Virais/Centro Médico Docente Assistencial/Escola Babiana de Medicina e Saúde Pública

\begin{tabular}{|c|c|c|c|c|c|c|c|}
\hline \multirow[b]{2}{*}{ Variáveis } & \multicolumn{2}{|c|}{ HTLV-1 ${ }^{-}$} & \multicolumn{2}{|c|}{ HTLV-1 ${ }^{+}$} & \multirow[b]{2}{*}{$\mathrm{OR}^{*}$} & \multirow[b]{2}{*}{ IC $95 \%$} & \multirow[b]{2}{*}{$\mathrm{p}$} \\
\hline & $\mathrm{n}^{0}$ & $\%$ & $\mathrm{n}^{0}$ & $\%$ & & & \\
\hline \multicolumn{8}{|l|}{ Idade da coitarca (anos) } \\
\hline$\leq 18$ & 28 & 43,8 & 44 & 69,8 & 2,98 & \multicolumn{2}{|c|}{$1,35-6,62<0,001$} \\
\hline$>18$ & 36 & 56,2 & 19 & 30,2 & & & \\
\hline \multicolumn{8}{|l|}{ Parceiros sexuais na vida $\left(\mathrm{n}^{\circ}\right)$} \\
\hline$>3$ & 8 & 12,1 & 30 & 46,9 & 6,40 & \multicolumn{2}{|c|}{$2,45-17,21<0,001$} \\
\hline$\leq 3$ & 58 & 87,9 & 34 & 53,1 & & & \\
\hline \multicolumn{8}{|l|}{ Práticas sexuais anais } \\
\hline $\operatorname{sim}$ & 12 & 18,8 & 23 & 36,5 & 2,49 & $1,03-6,08$ & 0,025 \\
\hline não & 52 & 81,2 & 40 & 63,5 & & & \\
\hline \multicolumn{8}{|l|}{ Uso de preservativos } \\
\hline freqüentemente (regular) & 11 & 17,2 & 7 & 11,1 & 1,66 & $0,54-5,18$ & 0,32 \\
\hline raramente/não (irregular) & 53 & 82,8 & 56 & 88,9 & & & \\
\hline \multicolumn{8}{|l|}{ História de DST } \\
\hline $\operatorname{sim}$ & 6 & 10,2 & 9 & 23,1 & 2,65 & $0,76-9,44$ & 0,08 \\
\hline não & 53 & 89,8 & 30 & 76,9 & & & \\
\hline \multicolumn{8}{|l|}{ História de transfusão sanguínea } \\
\hline $\operatorname{sim}$ & 5 & 7,7 & 16 & 26,7 & 4,36 & $1,36-14,84$ & $<0,01$ \\
\hline não & 60 & 92,3 & 44 & 73,3 & & & \\
\hline \multicolumn{8}{|l|}{ Uso de drogas ilícitas } \\
\hline $\operatorname{sim}$ & 1 & 1,5 & 1 & 1,6 & 1,03 & $0,01-82,2$ & $0,74^{*}$ \\
\hline não & 65 & 98,5 & 63 & 98,4 & & & \\
\hline
\end{tabular}

$\mathrm{OR}=$ odds ratio 
Tabela 4 - Resultados da regressão logística para fatores associados a soropositividade do HTLV-1 em mulberes.

\begin{tabular}{lcc}
\hline Variável & OR (IC 95\%) & Significância \\
\hline Práticas sexuais anais & $2,71(1,04-7,04)$ & 0,041 \\
Transfusão sanguínea & $5,19(1,58-16,98)$ & 0,007 \\
Número de parceiros sexuais na vida & $6,18(2,29-16,66)$ & $<0,001$ \\
Idade da coitarca & $2,61(1,12-6,08)$ & 0,027 \\
\hline Pseudo- $\mathrm{R}^{2}=0,304$ & & \\
\hline $\mathrm{OR}=$ odds ratio & &
\end{tabular}

\section{DISCUSSÃo}

Este estudo descreve e analisa características sociodemográficas, epidemiológicas e comportamentais de 64 pacientes com HTLV-1, comparando-as com mulheres soronegativas oriundas do mesmo serviço de atendimento, buscando reduzir diferenças relativas à seleção amostral, que foi não-aleatória. 0 perfil sociodemográfico entre os grupos de soronegativas e HTLV-1 positivas sem PET/MAH foi semelhante. As diferenças encontradas entre os três grupos em relação à escolaridade $(\mathrm{p}=0,05)$, estado civil ( $\mathrm{p}=0,012)$ e idade $(\mathrm{p}=0,007)$ podem ser atribuídas ao grupo de mulheres sintomáticas. Neste grupo, $63 \%$ das pacientes tinham idade superior a 50 anos, enquanto este percentual foi de $29,7 \%$ e $30,3 \%$, respectivamente, entre as soropositivas assintomáticas (sem PET/MAH) e soronegativas. Este achado pode ser explicado pelo longo tempo de infecção necessário, na maioria das vezes, para o desenvolvimento da sintomatologia e também na mudança do perfil dos pacientes encaminhados ao centro de referência, inicialmente caracterizados pela presença de alguma sintomatologia, portanto com uma idade maior, e, mais recentemente, oriundos da triagem sorológica em bancos de sangue e em gestantes, portanto mulheres mais jovens.

De acordo com estudos prévios, a baixa escolaridade está associada com maior soroprevalência de HTLV- ${ }^{3418}$. Neste estudo, contudo, a menor escolaridade encontrada entre as pacientes sintomáticas, e não verificada nas soropositivas assintomáticas, provavelmente se explica pela influência da idade, maior entre as mulheres com HAM/TSP e no menor acesso à escola por parte deste grupo. As mulheres com idade mais avançada viveram a infância e adolescência numa época na qual a educação era mais restrita a grupos populacionais de melhores condições sócioeconômicas e do sexo masculino. A mediana de idade das mulheres sintomáticas em meados dos anos 80 seria de 36 anos, enquanto entre os outros grupos essas medianas seriam de 25 e 21,5 anos. Segundo Pereira, o acesso à escola pelas mulheres aumentou progressivamente desde a década de 80 , alcançando um nivelamento educacional entre os sexos até a quarta série do ensino fundamental na década de $90^{15}$.

A maior (29,6\%) proporção de viúvas entre as mulheres sintomáticas pode ser explicada também pela influência da idade. Segundo o IBGE, a mulher apresenta uma expectativa de vida superior a do homem, além de ter maior dificuldade de encontrar um companheiro com o avanço da idade ${ }^{8}$. Em relação à renda, não houve diferença entre os grupos, apesar de alguns estudos sugerirem a associação de menor renda com a infecção pelo
HTLV-1 ${ }^{3418}$. O fato de não encontrarmos essa associação poderia ser explicado pela técnica de amostragem utilizada e por todos os grupos serem oriundos do mesmo serviço. Além disso, poder-seia aventar a possibilidade de que as pacientes com menor nível socioeconômico não mantenham o acompanhamento regular.

A análise dos fatores epidemiológicos associados a soropositividade pelo HTLV-1 foi realizada comparando as mulheres soropositivas e soronegativas. A hemotransfusão foi um fator de risco significante para a transmissão do HTLV-1, principalmente se ocorreu antes de 1994, quando a triagem para HTLV se tornou obrigatória nos bancos de sangue brasileiros. Nesse estudo, as mulheres HTLV-1 positivas receberam mais hemotransfusão que as soronegativas ( $\mathrm{p}<0,01$; OR não ajustada: 4,36; IC95\%: 1,36-14,84), sendo que 81,2\% (13/16) das transfusões, entre as soropositivas, ocorreram antes de 1993.

0 número de parceiros sexuais na vida foi maior entre as soropositivas $(6,1 \pm 5,9 \times 2,3 \pm 1,8)$, o mesmo verificado quando o número de parceiros foi estratificado usando três parceiros como ponto de corte ( $\mathrm{p}<0,001$; OR não ajustada 6,40 ; IC95\%: 2,45 - 17,21). Estudos anteriores demonstram que o maior número de parceiros sexuais durante a vida é um fator de risco para a infecção pelo HTLV-1, sendo mais desfavorável às mulheres que aos homens ${ }^{131019}$. A iniciação sexual mais precoce pode aumentar o risco de adquirir DST, sendo fator de risco também para a infecção pelo HTLV-1. A coitarca antes dos 18 anos foi mais comum entre as soropositivas ( $\mathrm{p}<0,001$; OR não ajustada: 2,98; IC95\%: 1,35-6,62). Além disso, as mulheres sintomáticas referem ter tido maior número de DST, sendo a sífilis a mais (6,34\%) freqüiente. Contudo, a grande quantidade de respostas indefinidas a esta questão prejudicou a análise deste fator de risco neste estudo. Apesar disso, um estudo prévio considera o diagnóstico de sífilis como fator de risco para a aquisição da infecção por HTLV-1 ${ }^{13}$. Os intercursos receptivos anais e vaginais são fatores de risco para infecção do HIV ${ }^{7}$. Neste estudo, a referência a práticas do sexo anal foi significativamente mais frequiente entre as pacientes soropositivas ( $\mathrm{p}=0,025$; OR não ajustada: 2,49; IC95\%: 1,03-6,08). Supõe-se que o intercurso anal seja mais traumático e, desta forma, representa uma maior possibilidade de transmissão do vírus, fato que é confirmado em relação ao $\mathrm{HIV}^{7}$. Contudo, em estudo realizado entre prostitutas do Zaire, não se verificou associação entre o tipo de intercurso e a soroprevalência do HTLV- ${ }^{19}$.

0 uso de drogas não foi um fator verificado entre as mulheres desta amostra. A técnica de amostragem utilizada e o número pequeno de mulheres participantes não permitem qualquer tipo de extrapolação para o que ocorre na população de mulheres infectadas. Mesmo assim, estudos em mulheres com HIV encontram uma menor proporção de UDI entre as mulheres. Finalmente, na análise multivariada, o modelo final teve uma predição de $71,4 \%$, mantendo a significância para a hemotransfusão, mais de três parceiros sexuais na vida, idade da coitarca antes dos 18 anos e presença de práticas sexuais anais, com intervalos de confiança não muito amplos, confirmando a presença dos principais fatores de risco entre as mulheres infectadas. 
Embora 0 aleitamento materno não tenha se mostrado como fator de risco importante em Salvador, suportando estudos prévios, sabe-se que esta via tem papel importante na transmissão da infecção pelo HTLV- ${ }^{3}$. Levando em consideração estes dados, recomenda-se que políticas públicas sejam reforçadas não só em relação à transmissão sexual como também em relação ao aleitamento materno. Recomendamos, por fim que a inclusão de teste de triagem no pré-natal em Salvador, seja mandatória.

\section{AGRADECIMENTOS}

Ao Dr. Ney Boa Sorte que contribuiu, igualmente, para a realização deste trabalho, que é parte da dissertação de Mestrado de Ivanoska Moxotó no Curso de Pós-graduação de Medicina e Saúde Humana da Escola Bahiana de Medicina e Saúde Pública.

\section{REFERÊNCIAS}

1. Carneiro-Proietti AB, Catalan-Soares BC, Castro-Costa CM, Murphy EL, Sabino EC, Hisada M, Galvão-Castro B, Alcantara LC, Remondegui C, Verdonck K, Proietti FA. HTLV in the Americas: challenges and perspectives. Revista Panamericana Salud Publica 19:44-53, 2006

2. Catovsky D, Greaves MF, Rose M, Galton DA, Goolden AW, McCluskey DR, White JM, Lampert I, Bourikas G, Ireland R, Brownell AI, Bridges JM, Blattner WA, Gallo RC. Adult T-cell lymphoma/leukemia in blacks from the west Indies. Lancet 1: 639-643, 1982.

3. Dourado I, Alcantara LC, Barreto ML, Teixeira MG, Galvão-Castro B. HTLV-I in the general population of Salvador, Brazil: a city with African ethnic and sociodemographic characteristics. Journal of Acquired Immune Deficiency Syndromes 34:527-531, 2003.

4. Edlich RF, Arnette JA, Williams FM. Global epidemic of human T-cell lymphotropic virus type-I (HTLV-I). Journal of Emergency Medicine 18:109-119, 2000.

5. Galvão-Castro B, Loures L, Rodriques LG, Sereno A, Ferreira Júnior OC, Franco LG, Muller M, Sampaio DA, Santana A, Passos LM, Proietti F. Distribution of human T-lymphotropic virus type I among blood donors: a nationwide Brazilian study. Transfusion 37:242-243, 1997.

6. Gessain A, Barin E, Vernant JC, Gout 0, Maurs L, Calender A. Antibodies to human t-lymphotropic virus type I in patients with tropical spastic paraparesis. Lancet 2:407-410, 1985

7. Greenblat RM, Hessol NA. Epidemiology and natural history of HIV infection in women. In: Anderson J (ed) A guide to the clinical care of women with HIV. Preliminary Edition, US Government Printing Office, Washington DC, p.1-33, 2000.
8. Instituto Brasileiro de Geografia e Estatísticas. Censo demográfico 2000Pesquisa Nacional em Domicílios; Nupcialidade e Fecundidade. Disponível em: httpp://www.ibge.gov.br/home/estatistica/população/censo2000/nupcialidadefecundidade/censo2000. Acessado em 20 de julho de 2003.

9. Kitagawa T, Fujishita M, Taguchi H, Miyoshi I, Tadokoro H. Antibodies to HTLV-I in Japanese immigrants in Brazil. Journal of the American Medical Association 256: 2342,1986

10. Larsen O, Sören A, Silva Z, Hedegaard K, Sandström A, Nauclér A, Dias F, Melbye M, Aaby P. Prevalence of HTLV-1 infection and associated risk determinants in an urban population in Guinea-Bissau, West África. Journal of Acquired Immune Deficiency Syndromes 25:157-163, 2000.

11. Mochizuki M, Watanabe T, Yamaguchi K, Takatsuki K, Shirao M, Yoshimura K, Nakashima S, Mori S, Araki S, Miyata N. HTLV-1 uveitis: a distinct clinical entity caused by HTLV-1. Japanese Journal of Cancer Research 83:236-239, 1992.

12. Mueller N. The epidemiology of HTLV-I infection. Cancer Causes Control 2:37-52, 1991

13. Murphy EL, Figueroa JP, Gibbs WN, Brathwaite A, Holding-Cobham M, Waters D, Cranston B, Hanchard B, Blattner WA. Sexual transmission of Human Tlymphotropic virus type I (HTLV-I). Annals of Internal Medicine 111:555-560, 1989.

14. Osame M, Matsumoto M, Usuku K, Izumo S, Ijichi N, Amitani H, Tara M, Igata A. HTLV-1 associated myelopathy, a new clinical entity. Lancet 1:1031$1032,1986$.

15. Pereira TI. Educação e mercado de trabalho no contexto feminino. Disponível em: URL: http:// www.portalsociologia.hpg.ig.com.br/educ_merctrabfem.htm. Acesso em 03/04/2005.

16. Poiesz BJ, Ruscetti FW, Gazdar AF, Bunn PA, Minna JD, Gallo RC. Detection and isolation of type-C retrovirus particles form fresh and cultured lymphocytes of a patient with cutaneous t-cell lymphoma. Proceedings of the National Academy of Sciences of the United States of America 77:7415-7419, 1980.

17. Proietti FA, Carneiro-Proietti ACF, Catalan-Soares BC, Murphy EL. Global epidemiology of HTLV-1 infection and associated diseases. Oncogene.24:6058$6068,2005$.

18. Rouet F, Herrmann-Storck C, Courouble G, Deloumeaux J, Madani D, Strobel M. A case-control study of risk factors associated with human T-cell lymphotrophic virus type-I seropositivity in blood donors from Guadeloupe, French West Indies. Vox Sanguinis 82:61-66, 2002.

19. Wiktor SZ, Piot P, Mann JM, Nzilambi NH, Vercauteren G, Blattner WA, Quinn TC. Human T cell lymphotropic virus type I (HTLV-I) among Female Prostitutes in Kinshasa, Zaire. Journal of Infectious Diseases 161:1073-1077, 1990.

20. Yoshida M, Miyoshi I, Hinuma Y. Isolation and characterization of retrovirus from cells lines of human adult T-cell leukemia and its implications in the disease. Proceedings of the National Academy of Sciences of the United States of America 79:2031-2035, 1982.

21. Nunes CL, Gonçalves LA, Silva PT, Bina JG. Clinical-epidemiological characteristics of a group of HIV/AIDS infected women in Salvador-Bahia Revista da Sociedade Brasileira de Medicina Tropical 37:436-440,2004. 